\title{
ESTRATÉGIAS PARA O ALCANCE DE OBJETIVOS AFETIVOS NO ENSINO REMOTO
}

Antonio Carlos $\mathrm{Gil}^{1}$, Arquimedes Pessoni ${ }^{2}$

\section{RESUMO}

O trabalho ora apresentado foi motivado pela ampla utilização da videoconferência como alternativa às aulas presenciais em decorrência da pandemia provocada pela Covid-19. Pode ser caracterizado como um ensaio teórico que tem como proposta discutir a eficácia do ensino remoto para o alcance de objetivos afetivos de aprendizagem. Os objetivos mais facilmente alcançáveis mediante aulas expositivas - e consequentemente mediante videoconferências - são os cognitivos, notadamente nos níveis de memorização e compreensão. Quando os objetivos de aprendizagem estão no domínio afetivo - que envolvem emoções, sentimentos e atitudes - outras estratégias são requeridas. Assim, com base na contribuição de teóricos no campo dos objetivos de aprendizagem e nos resultados de pesquisas empíricas, procede-se à discussão das possibilidades de utilização de diferentes estratégias mediante plataformas de cursos on-line. São discutidas as estratégias de discussão reflexiva, trabalho colaborativo, grupo de verbalização/grupo de observação, tutoria por pares, dramatização, simpósio, seminário e gamificação. $O$ estudo aponta para a adoção do ensino híbrido como forma preferencial de ensino superior no cenário póspandemia, mas que deve vir acompanhado da capacitação dos docentes na utilização de estratégias de ensino grupal.

Palavras-chave: Objetivos afetivos. Ensino remoto. Ensino híbrido. Pandemia. Covid-19.

\section{Como citar este documento - ABNT}

GIL, Antonio Carlos; PESSONI, Arquimedes. Estratégias para o alcance de objetivos afetivos no ensino remoto. Revista Docência do Ensino Superior, Belo Horizonte, v. 10, e024493, p. 1-18, 2020. DOI: https://doi.org/10.35699/22375864.2020.24493.

Recebido em: 10/08/2020 Aprovado em: 16/10/2020 Publicado em: 28/11/2020

\footnotetext{
${ }^{1}$ Universidade Municipal de São Caetano do Sul (USCS), São Caetano do Sul, SP, Brasil. ORCID ID: https://orcid.org/0000-0002-4967-8280. E-mail: acgil@uol.com.br.

2 Universidade Municipal de São Caetano do Sul (USCS), São Caetano do Sul, SP, Brasil.

ORCID ID: https://orcid.org/0000-0003-1050-3405. E-mail: arquimedes.pessoni@uol.com.br.
} 


\section{ESTRATEGIAS PARA LOGRAR OBJETIVOS AFECTIVOS EN LA EDUCACIÓN REMOTA}

\section{RESUMEN}

El trabajo aquí presentado fue motivado por la amplia utilización de la videoconferencia como alternativa a las clases presenciales debido a la pandemia provocada por Covid-19. Se puede caracterizar como un ensayo teórico que tiene como propuesta discutir la efectividad de la educación remota para lograr objetivos afectivos de aprendizaje. Los objetivos más fáciles de conseguir a través de clases expositivas $y$, en consecuencia, a través de videoconferencias, son los cognitivos, sobre todo en los niveles de memorización y comprensión. Cuando los objetivos de aprendizaje están en el dominio afectivo, que involucran emociones, sentimientos y actitudes, se requieren otras estrategias. Así, a partir del aporte de los teóricos en el campo de los objetivos de aprendizaje y de los resultados de investigaciones empíricas, se discuten las posibilidades de utilización de distintas estrategias en plataformas de cursos en línea. Se debaten las estrategias de discusión reflexiva, trabajo colaborativo, grupo de verbalización/grupo de observación, tutoría entre pares, dramatización, simposio, seminario y gamificación. El estudio apunta a la adopción de la educación híbrida como la forma preferencial de educación superior en el escenario pospandémico, pero que debe estar acompañada de la formación de los docentes en el uso de estrategias de enseñanza grupal.

Palabras clave: Objetivos afectivos. Enseñanza remota. Enseñanza híbrida. Pandemia. Covid19.

\section{STRATEGIES FOR ACHIEVING AFFECTIVE OBJECTIVES IN REMOTE EDUCATION}

\section{ABSTRACT}

This work was motivated by the wide use of videoconferencing as an alternative to face-toface classes due to the pandemic caused by Covid-19. It can be characterized as a theoretical essay that aims to discuss the effectiveness of remote teaching to achieve affective learning objectives. The most easily achieved objectives through expository classes - and consequently through videoconferences - are cognitive, notably at the levels of memorization and understanding. When learning objectives are in the affective domain which involve emotions, feelings and attitudes - other strategies are required. Thus, based on the contribution of theorists in the field of learning objectives and the results of empirical research, the possibilities of using different strategies are discussed through online course platforms. Strategies for reflective discussion, collaborative work, verbalization group/observation group, peer tutoring, dramatization, symposium, seminar and gamification are discussed. The study points to the adoption of hybrid education as the preferred form of higher education in the post-pandemic scenario, but which must be accompanied by the training of teachers in the use of group teaching strategies.

Keywords: Affective goals. Remote teaching. Hybrid teaching. Pandemic. Covid-19. 


\section{INTRODUÇÃO}

O planejamento do ensino requer o esclarecimento acerca das principais decisões a serem tomadas ao longo do processo, o que envolve a formulação de objetivos, a determinação dos conteúdos, a seleção de estratégias e recursos de ensino e a avaliação da aprendizagem. A formulação dos objetivos constitui a principal dentre estas decisões, pois é em torno delas que gravitam todas as outras atividades. Sem objetivos claros não há como determinar o conteúdo programático dos cursos. Também não há como determinar as estratégias e os recursos mais adequados para alcançá-los. E, se não forem suficientemente claros, não será possível verificar em que medida foram alcançados.

A importância atribuída aos objetivos levou autores como Bobbitt (1924), Tyler (1949), Bloom et al. (1956), Krathwohl; Bloom; Masia (1964), Mager (1962) e Anderson et al. (2001) a desenvolver trabalhos com o propósito explícito de instruir os educadores no sentido de elaborar objetivos de aprendizagem. Graças a esses trabalhos, a formulação de objetivos tornou-se elemento indispensável nos planos de ensino elaborados por professores em todos os níveis de ensino.

Um dos aspectos mais relevantes na elaboração de objetivos está em sua classificação. Foi movido por essa importância que Bloom et al. (1956) estabeleceram a taxonomia dos objetivos educacionais, que os classifica em cognitivos, afetivos e psicomotores. Esta taxonomia, caracterizada por ser um modelo, simples, claro e eficaz, fornece uma estrutura para planejar, executar e avaliar a eficácia das atividades educacionais.

O domínio cognitivo abrange os objetivos relacionados a conhecimentos ou capacidades intelectuais. O domínio afetivo abrange aqueles relacionados a sentimentos, opiniões e atitudes. O domínio psicomotor, por fim, abrange os objetivos relacionados a atividades de natureza neuromuscular.

Com a especificação dos objetivos que pretendem alcançar, os professores podem definir com mais propriedade as estratégias a serem adotadas. Assim, quando formulam objetivos cognitivos em seus níveis mais elementares - como memorização e compreensão podem selecionar leituras e aulas expositivas como estratégias adequadas. Se, porém, seus objetivos estiverem em níveis mais elevados do domínio afetivo - como síntese e avaliação - , a discussão pode ser mais recomendada. Se formularem objetivos afetivos, dentre as estratégias mais recomendadas estão a discussão e a dramatização. Se, porém, os objetivos estiverem no domínio psicomotor, a demonstração poderá mostrar-se uma estratégia adequada.

Quando o ensino é ministrado presencialmente em sala de aula, os professores podem direcionar suas atividades com vistas a atingir os objetivos pretendidos. A aula presencial que é uma das estratégias mais tradicionais - pode ser adotada com o intuito de favorecer 
nos estudantes a memorização e a compreensão dos conceitos, resultados de natureza cognitiva. Mas se o professor espera que os estudantes procedam a uma avaliação crítica dos conteúdos ministrados, pode promover, ainda em sala de aula, diferentes modalidades de discussão. Por outro lado, se o que pretende é o alcance de objetivos afetivos, o indicado seria promover dramatizações. Por fim, se pretende alcançar objetivos psicomotores, pode proceder a demonstrações.

As aulas presenciais - embora insuficientes para o alcance de muitos objetivos de aprendizagem por serem tradicionais, de fácil implementação, acessíveis à maioria dos professores, econômicas e bastante flexíveis - ainda constituem uma das estratégias didáticas mais utilizadas nos diferentes níveis de ensino. Porém, em decorrência das restrições de distanciamento social, determinadas pela pandemia da Covid-19, passaram a ser substituídas pelo ensino remoto na maioria dos estabelecimentos de ensino.

A intensa utilização do ensino remoto, de forma evidentemente não planejada, por sua vez, vem determinando múltiplas discussões acerca de sua eficácia. Alguns benefícios são evidentes. Além de diminuir as aglomerações, o ensino remoto proporciona aos estudantes menores gastos com deslocamento e alimentação, podendo garantir-lhes menos cansaço e mais disposição para assistir às aulas. Mas a adoção do ensino remoto também apresenta desvantagens. Os estudantes podem ter dificuldade para manusear computadores e internet fora da escola e para se manterem atentos durante as aulas. Além disso, os professores podem não ter as habilidades necessárias para utilizar adequadamente a tecnologia do ensino remoto. As instituições de ensino, por sua vez, podem carecer da infraestrutura necessária para garantir um ensino de qualidade.

Mas a grande questão determinada por este quadro é a do quanto o ensino remoto é eficaz para possibilitar aos estudantes a assimilação dos conteúdos propostos e a capacitação para sua aplicação na vida social e profissional. Ou, em outras palavras, se são suficientes para garantir o alcance dos objetivos de aprendizagem propostos nos planos de ensino.

A resposta a esta questão é relativamente simples quando se considera o domínio cognitivo. Como este se refere a capacidades intelectuais como memorização, compreensão, aplicação, análise e avaliação, as aulas expositivas, ainda que apresentadas remotamente, associadas a leituras e a exercícios estruturados, podem mostrar-se suficientes para o seu alcance.

Quando, porém, se consideram objetivos psicomotores, a resposta não parece tão simples. Os objetivos psicomotores, por se referirem a atividades que envolvem habilidades relacionadas à manipulação de máquinas, instrumentos ou objetos, são mais difíceis de serem alcançados com o ensino remoto. 
E quando se consideram os objetivos afetivos? Como estes se referem a fenômenos como motivação, sentimentos, emoções, disposições e valores, cabe indagar até que ponto uma modalidade de ensino em que professores e estudantes permanecem a distância, sem qualquer contato físico ou relacionamento social direto, é capaz de garantir o alcance de objetivos nesse domínio. Assim, foi elaborado o presente trabalho, que tem como proposta discutir a eficácia do ensino remoto no alcance dos objetivos afetivos. Trata-se de um ensaio teórico fundamentado na contribuição de autores que tratam da formulação de objetivos educacionais. Seu objetivo é, pois, refletir criticamente acerca das estratégias e das tecnologias que podem ser adotadas com vistas ao alcance de objetivos instrucionais mediante atividades educativas desenvolvidas remotamente. Espera-se que contribua para orientar professores do ensino superior das diferentes áreas na seleção de estratégias para apoiar o ensino remoto - não apenas durante a pandemia da Covid-19, já que se prevê a intensificação do ensino híbrido, que requer o desenvolvimento de atividades desenvolvidas a distância.

\section{O DOMÍNIO AFETIVO}

Os objetivos correspondentes ao domínio afetivo enfatizam um sentimento, uma emoção ou um certo grau de aceitação ou rejeição. Esses objetivos, que variam desde a simples atenção a fatos e comportamentos complexos, são classificados em cinco níveis: 1) receptividade, ou disposição para tomar consciência de um fato e prestar atenção a ele; 2) resposta, que indica disposição para receber o estímulo dado, não o evitando; 3) valorização, ou seja, o reconhecimento do valor de alguma coisa; 4) organização, que envolve o relacionamento de um valor novo aos que são aceitos; e 5) caracterização por um valor ou complexo de valores, que significa agir consistentemente de acordo com os valores internalizados (KRATHWOHL; BLOOM; MASIA, 1964).

Fica evidente que essa taxonomia é ordenada de acordo com o princípio da internalização, que se refere ao processo pelo qual o afeto de uma pessoa em relação a um objeto passa de um nível de consciência geral para um ponto em que este é internalizado e passa a orientar ou controlar consistentemente o comportamento da pessoa (SEELS; GLASGOW, 1990).

Constata-se, porém, que o domínio afetivo tem sido tratado de maneira muito mais ampla. Fasso e Knight (2018), mediante ampla revisão da literatura, demonstram como esse conceito tem sido utilizado com outros significados além dos propostos pelos criadores da taxonomia. Dentre outros significados, foram identificados: atitudes em relação ao curso (BAKER et al., 2010); emoções na aprendizagem (ECCLESTONE; HAYES, 2008); engajamento cognitivo (JAGGER, 2013); ideias, valores, atitudes e crenças orientações e ideologias, emoções e sentimentos no aprendizado (BIRBECK, 2009); automotivação, atitudes e sentimentos em relação ao comportamento como profissional (OLATUNJI, 2013); autoconceito, moralidade, saúde mental e personalidade (MARTIN; BRIGGS, 1986); 
dimensão interpessoal do aprendizado (SHOFFNER, 2009); cortesia, respeito, lidar com emoções, comunicação, preconceitos, responsabilidade e engajamento (CATE; DE HAES, 2000); moral e decisões éticas (LYNCH; HART; COSTA, 2014); engajamento e autoeficácia (FALOUT; ELWOOD; HOOD, 2009); tédio, frustração, confusão e prazer (D’MELLO; GRAESSER, 2012); empatia (DU BOULAY, 2013); relações humanas, autoconsciência, habilidades de liderança, habilidades de construção de equipes e socialização (CLEVELAND-INNES; ALLY, 2007); disposição individual, vontade e preferências (GROUNLUND, 1995), autoeficácia, interação social, emoções e conexão com a terra (VAN DER HOEVEN KRAFT et al., 2011), emoções, empatia e humor (SNELSON; ELISON-BOWERS, 2009).

De fato, o domínio afetivo é muito mais complexo que o cognitivo. Tanto é que a obra que apresenta a taxonomia desse domínio (KRATHWOHL; BLOOM; MASIA, 1964) só foi publicada oito anos após o primeiro volume, que tratava do domínio cognitivo. Isso se explica, em parte, pelo fato de o domínio afetivo ter sido considerado durante muito tempo como inferior ao cognitivo. Afinal, a escola tem sido considerada tradicionalmente o local adequado para a aquisição de conhecimentos. A afetividade foi vista, por muito tempo, como algo a ser aprendido mais apropriadamente no âmbito de outras instituições sociais, como a família, embora se constate um interesse crescente pelos temas relacionados à afetividade na educação (BOLIN; KHRAMTSOVA; SAARNIO, 2005; AKOS; KRETCHMAR, 2017).

Requer-se, pois, uma revisão da taxonomia dos objetivos educacionais referentes ao domínio afetivo, como já ocorreu com o domínio cognitivo (KRATHWOHL; BLOOM; MASIA, 1964; ANDERSON et al., 2001). Requer-se uma taxonomia capaz de avançar além da caracterização por um valor ou complexo de valores, que esclareça acerca da formulação de objetivos referentes a sentimentos, emoções, atitudes, crenças, valores, crescimento pessoal etc. Mas enquanto se aguarda essa revisão, os educadores continuam formulando objetivos relacionados a esses tópicos.

A complexidade do domínio afetivo leva à compreensão que os objetivos afetivos são os mais difíceis de alcançar nos programas educativos. E de uma maneira muito especial na educação a distância, os relacionamentos interpessoais e, consequentemente, a afetividade, tendem a ser minimizados. Isso tem levado alguns autores a criticar essa modalidade de ensino. Dentre essas críticas, cabe ressaltar as que são fundamentadas na Teoria da Presença Social (SHORT; WILLIAMS; CHRISTIE, 1976). Essa teoria estabelece que tal fenômeno é uma qualidade do próprio meio de comunicação, sugerindo que eles variam em seu grau de presença social e que essas variações são importantes para determinar como os indivíduos interagem. A capacidade desses meios para transmitir informações acerca da expressão facial e pistas não verbais contribuiria para ampliar essa presença social. Assim, quando as informações são apresentadas de maneira tal que haja esse aumento, tendem a ser mais lembradas, tornando a aprendizagem mais envolvente (HOMER; PLASS; BLAKE, 2008). 
Tratando especificamente da educação a distância, Lee (2000) constata que os alunos online costumam relatar falta de contato social e sentimentos de isolamento em relação a seus professores e colegas. Dessa forma, a natureza assíncrona do aprendizado on-line criaria incerteza entre os alunos em decorrência da ausência ou atraso no feedback do professor, o que poderia levar à incerteza e à ansiedade.

Mas essas críticas feitas à educação a distância não consideram apropriadamente as tecnologias - já de quinta geração - que vêm sendo incorporadas a essa modalidade educativa. Considere-se, por exemplo, o significado da plataforma Watson, que foi pensada para ser capaz de obter informações produzidas por seres humanos e que utiliza a linguagem natural, incorporando contextos, referências ambíguas, noções implícitas e outros componentes socioculturais. Outro exemplo é o robô Pepper, que consegue memorizar os rostos dos alunos, registrar seu comportamento e informar o professor acerca do que estão repetindo em sala de aula.

Em apoio à educação a distância, cabe, também, considerar a Teoria da Distância Transacional, formulada por Moore (1997), segundo a qual a educação a distância não se caracteriza simplesmente pela separação geográfica entre professores e alunos, mas por um universo de relações que se dão quando eles estão separados no espaço e/ou no tempo. Esta separação conduz a padrões especiais de relações entre professores e alunos. Com ela, surge um espaço psicológico e comunicacional, que Moore (1997) denomina "distância transacional". Esta distância precisa ser ultrapassada por professores, alunos e instituições que promovem a educação a distância para que ocorra um processo de aprendizagem eficaz, deliberado e planejado. Assim, três componentes devem ser trabalhados nos programas de educação a distância para encurtar a distância transacional: provisão de diálogo entre professores e alunos, estrutura dos programas e autonomia dos estudantes.

\section{OBJETIVOS E ESTRATÉGIAS DE ENSINO}

A identificação do domínio da aprendizagem é essencial na determinação da estratégia de ensino. Considere-se, por exemplo, que em curso na área da Educação pretende-se abordar o tópico "feedback". Um objetivo poderia ser "caracterizar diferentes modalidades de feedback". Outro objetivo poderia ser "valorizar a utilização do feedback em sala de aula". 0 primeiro insere-se no domínio cognitivo e o segundo, no afetivo. Assim, para o alcance do primeiro objetivo, aulas expositivas ou leituras seriam estratégias adequadas. Já para o alcance do segundo objetivo, seria recomendado adotar outras estratégias, como discussão em grupo ou dramatização.

A vinculação da estratégia de ensino aos objetivos de aprendizagem é questão de fundamental importância quando se trata de ensino remoto. Isto porque o que se verifica em tempos de pandemia é que a base do ensino passou a ser a aula oferecida a distância. 0 
professor, que habitualmente ministrava aulas expositivas, passou a oferecê-las remotamente, valendo-se, inclusive, dos recursos auxiliares proporcionados por tecnologias de apresentação, como o PowerPoint.

O sucesso dessa transição para o ensino remoto depende de diversos fatores, como disposição dos estudantes, capacitação do professor, qualidade da tecnologia, objetivos de aprendizagem e estratégias de ensino. O professor precisa ter o domínio do assunto e a competência para lidar com as novas tecnologias. Os estudantes também precisam dominálas e sentir-se dispostos a aprender a distância. A tecnologia, por sua vez, precisa ser de qualidade para garantir que o processo se efetive. Os objetivos de aprendizagem e as estratégias de ensino, por fim, precisam estar harmonizadas para que os resultados sejam favoráveis.

Cabe considerar, todavia, que essa situação não corresponde, a rigor, ao que se denomina ensino a distância, mas sim ao ensino remoto, o que faz uma grande diferença. $O$ ensino a distância - assim como o ensino híbrido, que junta elementos tanto do ensino presencial quanto do ensino a distância - é efetivamente planejado como tal (MOORE; KEARSLEY, 2013; HORN; STAKER, 2015). As aulas são geralmente gravadas em estúdios, com apoio de pessoal técnico, e posteriormente editadas. Os estudantes matriculados nessa modalidade de ensino apresentam características que os distinguem dos matriculados em cursos presenciais, tanto em relação à faixa etária e nível socioeconômico quanto à motivação para estudar e às expectativas profissionais. Já os estudantes que neste momento estão tendo aulas remotamente não escolheram essa modalidade, embora seja possível que muitos, em decorrência da experiência, possam aderir ao ensino a distância. Da mesma maneira, é possível que as instituições de ensino passem a considerar a possibilidade de adotar alguma forma de ensino híbrido como a preferencial na oferta de cursos futuros.

A rigor, a questão que se apresenta refere-se não ao ensino remoto, mas às aulas on-line oferecidas na modalidade síncrona, ou seja, aulas que ocorrem em tempo real, através de plataformas de videoconferência (NORTHEY et al., 2015; MARTIN; PARKER, 2014). Trata-se, portanto, de uma questão a ser considerada principalmente em relação ao ensino híbrido, que é desenvolvido segundo diferentes modelos (HORN; STAKER, 2015), um deles sendo o da aula invertida (BERGMANN, 2016).

Um dos problemas a se considerar nesta nova realidade está na estratégia adotada pelo professor para promover o ensino remoto. Assim, cabe acatar o que tradicionalmente tem ocorrido com o ensino, sobretudo no nível superior. A estratégia mais adotada tem sido a aula expositiva, que, como já foi considerado, é bastante flexível e, consequentemente, aplicável para o alcance de muitos objetivos educacionais. Mas, como também já foi lembrado, ela não é adequada para todos os objetivos. Ora, o professor que, de um momento para outro, sem aviso prévio, foi solicitado a ministrar aulas remotamente, 
buscou, naturalmente, adaptar as aulas que seriam dadas em classe à modalidade virtual. Assim, desde que capacitado quanto ao conteúdo e aos meios técnicos de comunicação, poderá sentir-se em condições de proporcionar um ensino eficaz. Mas terá que levar em consideração em que medida as estratégias adotadas são coerentes com os objetivos de aprendizagem formulados para sua disciplina, de modo especial nas situações em que o professor almeja o alcance daqueles do domínio afetivo.

O professor, desde que tenha clareza acerca do significado de sua disciplina no contexto de curso em que leciona, das competências e dos interesses de seus estudantes, é capaz de elaborar seus objetivos, que correspondem àquilo que ele espera que eles sejam capazes de saber, reconhecer ou fazer. Ou seja, espera que os discentes sejam capazes de formular objetivos cognitivos, afetivos e psicomotores.

Esses objetivos, para que sejam úteis, devem orientar-se para o que os estudantes serão capazes de fazer. Devem ser relevantes para os propósitos da disciplina e do curso. E o que é mais relevante no contexto deste trabalho é que tais objetivos devem vincular-se a uma estratégia que facilite seu alcance. O termo estratégia é aqui utilizado num sentido abrangente, referindo-se aos métodos, às técnicas e a outros procedimentos adotados para facilitar o processo de aprendizagem.

A seleção da estratégia deve, pois, levar em consideração os domínios da aprendizagem. Assim, no domínio cognitivo, em seus níveis inferiores (memorização, compreensão e aplicação), uma das estratégias mais utilizadas é a aula expositiva, que se mostra adequada para proporcionar uma visão geral de um tópico específico ou para aprofundar aspectos importantes de cada um deles. Palestras e conferências apresentadas por especialistas, assim como vídeos alusivos a conteúdos específicos, também são estratégias recomendadas. Podem, ainda, ser consideradas algumas formas de aprendizado individualizado, como leituras específicas, estudos mediados por computador e exercícios estruturados.

Nos níveis mais elevados do domínio cognitivo (análise, avaliação e criação), a discussão em classe é uma das estratégias mais adotadas. Além da discussão em sua modalidade mais clássica, podem ser adotadas a discussão desenvolvente, em que o professor quebra as questões em partes e faz com que todos os estudantes trabalhem com uma parte ao mesmo tempo, e a discussão socrática, em que os estudantes desempenham um papel mais ativo. Outras estratégias adequadas neste nível são jogos, simulações e estudos de caso.

No domínio afetivo, as estratégias mais adequadas são as que se mostram capazes de promover o envolvimento dos estudantes nas tarefas e promover mudanças de interesses, atitudes e valores. A discussão reflexiva é uma das mais clássicas estratégias adotadas com o propósito de alcançar objetivos nesse domínio. Outras estratégias que podem ser utilizadas são painéis, seminários, jogos cooperativos e dramatização. 
No domínio psicomotor, as estratégias mais adequadas são as que contribuem para o desenvolvimento de habilidades motoras. A demonstração é estratégia clássica adotada nesse domínio. Muitas das atividades são desenvolvidas não em salas de aula convencionais, mas em laboratórios.

\section{ESTRATÉGIAS ADEQUADAS PARA O ALCANCE DE OBJETIVOS AFETIVOS}

Objetivos afetivos são provavelmente os mais difíceis de alcançar. As aulas expositivas, que ainda constituem uma das estratégias mais adotadas no ensino superior, por si só, são insuficientes para o alcance de objetivos nesse domínio. Assim, em situações de ensino presencial, recomenda-se que, na sequência às aulas expositivas sejam adotadas outras estratégias, como a apresentação de um vídeo, uma discussão em classe, a apresentação de situações da vida real, uma dramatização, um seminário ou um jogo. Já no ensino remoto, os professores têm utilizado principalmente a videoconferência, que também se mostra insuficiente para o alcance de objetivos afetivos. Recomenda-se, portanto, que os professores, na sequência, valham-se de outras estratégias que, assim como a videoconferência, possam ser utilizadas remotamente e capazes de promover mudanças afetivas nos estudantes. Dentre estas estratégias estão:

\section{Discussão reflexiva}

A discussão reflexiva é uma das estratégias mais utilizadas para o alcance de objetivos cognitivos em nível mais elevado, mas também dos afetivos referentes ao reconhecimento do valor de uma coisa, um fenômeno ou um comportamento. Nessa modalidade de discussão, o professor elabora algumas questões referentes a determinado tópico para as quais não existe uma única resposta adequada e propõe aos estudantes para que reflitam a respeito e verbalizem suas opiniões.

Para ser mais efetiva, a discussão reflexiva deve ser realizada de forma síncrona. 0 professor, para conduzi-la eficazmente, precisa abster-se da expressão de suas próprias opiniões e estimular os estudantes a participar. Dessa maneira, evita que o envolvimento fique limitado a poucos alunos, pois é uma modalidade de discussão que exige bastante habilidade de professor, que precisa saber ouvir, tratar com apreço os estudantes, reforçar a participação, atentar para o tempo e evitar o consenso prematuro dos discentes. Há estudos que indicam que as discussões, mesmo na modalidade assíncrona, podem levar a um aumento da ansiedade dos alunos e à disposição para abandoná-la (GRIFFIN; ROY, 2019).

Como a discussão reflexiva não tem como propósito a aquisição de novos conhecimentos, mas sim a reflexão acerca do que já se sabe, deve ocorrer preferencialmente após a conclusão de outras atividades, tais como uma exposição, leitura de um texto ou 
visualização de um vídeo. As classes, por sua vez, não podem ser muito numerosas para possibilitar que todos os estudantes participem da discussão.

\section{Trabalho colaborativo}

Nesta estratégia, os estudantes trabalham em conjunto para desempenhar diferentes tipos de atividades, como análise, avaliação ou desenvolvimento de um projeto. Ela requer que os participantes colaborem entre si, ouçam uns aos outros, argumentem e negociem com seus colegas. É, pois, uma estratégia que contribui para aprimorar relações interpessoais, estimular mudanças atitudinais e ampliar a capacidade de enxergar as coisas sob a perspectiva do outro (JOHNSON; JOHNSON, 1999).

O trabalho colaborativo pode ser utilizado, por exemplo, dividindo a classe em pequenos grupos (reunidos em salas virtuais) para que marquem uma posição em relação a um projeto ou política educacional. Para que se efetive, o trabalho colaborativo precisa valer-se de múltiplos suportes, tais como videoconferência associada a fóruns, chats e e-mails.

\section{Grupo de Verbalização/Grupo de Observação (GV/GO)}

Uma das mais tradicionais técnicas de dinâmica de grupo, o Grupo de Verbalização/Grupo de Observação, também pode ser aplicada com proveito em classes virtuais, desde que não sejam muito numerosas (WHITE, 1974). O professor divide a classe em dois grupos de igual tamanho. Propõe, então, um tema para discussão e pede para que os membros do primeiro grupo (GV) discutam-no durante cerca de 15 minutos. Pede aos membros do segundo grupo (GO) para que observem a participação dos estudantes envolvidos na discussão, indicando alguns aspectos a serem considerados. Após o término da discussão, estes são solicitados a avaliar o desempenho dos membros do primeiro grupo. Ao final, o professor promove um desfecho para a sessão, ressaltando aspectos que julga importantes.

\section{Tutoria por pares}

Na tutoria por pares, participantes monitoram e apoiam uns aos outros. Trata-se, portanto, de uma estratégia que estimula o pensamento crítico e a reflexão, contribui para o desenvolvimento de habilidades interpessoais e estimula mudanças atitudinais (GREENWOOD; CARTA; HALL, 1988). Os estudantes têm a oportunidade de aprender com o trabalho dos outros e de exercitar a tutoria. Por exemplo, cada estudante pode ser solicitado a rever o trabalho desenvolvido por outro colega e oferecer sugestões ou aperfeiçoá-lo de acordo com princípios aprendidos durante o curso. O formato mais adequado para a tutoria por pares é o de fórum de discussão. 


\section{Dramatização}

A dramatização, que tem seus fundamentos na teoria dos papeis, desenvolvida por Moreno (1983), é uma estratégia utilizada para desenvolver habilidades interpessoais mediante o desempenho de atividades semelhantes às que seriam desenvolvidas na vida real. É possível simular on-line situações como uma entrevista de seleção, uma acareação policial, uma venda de produtos, uma orientação para tomada de cuidados com a saúde ou, até mesmo, uma negociação para praticar sexo seguro.

Considere-se, por exemplo, a situação em que um profissional de saúde procura convencer um paciente a observar a prescrição de determinado medicamento. Os participantes são, então, divididos em grupos constituídos por duas pessoas, sendo que uma delas irá desempenhar o papel de profissional de saúde e a outra de paciente. O desempenho é feito no âmbito de classes virtuais. É possível, a critério de professor, definir diferentes perfis do paciente para facilitar a análise do comportamento do profissional em relação a pessoas com características diferentes.

Após a simulação, o professor fornece feedback aos participantes. É preciso garantir que ele seja suficientemente esclarecido e que seja muito mais descritivo do que avaliativo. Também é possível solicitar que alguns estudantes forneçam feedback. Neste caso, é preciso informar previamente em que aspectos deverão se atentar durante a simulação.

\section{Simpósio}

Nesta modalidade, algumas pessoas (de duas a cinco) com conhecimentos especializados em relação a determinado tema, apresentam-no sob determinado aspecto. Após as apresentações, o professor - que assume o papel de moderador - estimula os alunos a fazer perguntas aos especialistas (GIL, 2020). Para ser mais eficaz, deve ser realizado como videoconferência de forma síncrona.

\section{Seminário}

O seminário tem sido muito utilizado no ensino superior. Nem sempre, porém, é usado da maneira mais adequada, pois frequentemente é desenvolvido como uma exposição, apresentada por um estudante, seguida de debate não muito intenso posto que o apresentador foi o único solicitado a se preparar previamente. Mas, desde que bem conduzido, pode ser aplicado no ensino remoto, tanto para o alcance de objetivos cognitivos quanto afetivos (GIL, 2020).

Para que seja eficaz, o professor indica previamente um texto - ou um tema de estudo que deve ser lido e discutido por todos. Essa tarefa deve ser efetivada em pequenos grupos, 
mediante a constituição de classes virtuais. Cada grupo deverá escolher um coordenador para dirigir a discussão e um relator para apresentar as conclusões.

Como o que se pretende é alcançar objetivos afetivos, é necessário que o texto seja provocativo e que conduza a múltiplas interpretações. Assim, os participantes dos grupos devem ser solicitados a examinar o assunto sob diferentes perspectivas e levantar os problemas surgidos a partir de sua análise. Após a conclusão dessa etapa, todos os participantes se reúnem na sessão plenária. Assim, cada coordenador ou relator procede à apresentação das conclusões do grupo. O professor faz, então, a avaliação dos trabalhos dos grupos e elabora uma síntese desses trabalhos.

\section{Gamificação}

A gamificação constitui, provavelmente, a mais importante dentre as estratégias adotadas na educação a distância quando o propósito é o alcance de objetivos no domínio afetivo. Gamificação é aqui entendida como a estratégia que utiliza mecanismos de jogos, tais como competições, premiações e pontos, em ambientes fora do contexto dos jogos, com o propósito explícito de motivar os estudantes.

Os benefícios da gamificação para o alcance de objetivos afetivos são bem evidentes: 1) promove o envolvimento dos estudantes; 2 ) proporciona a criação de um ambiente imersivo e interativo; 3) torna o processo de aprendizagem mais leve e agradável; 4) cria um sentimento de competição entre os estudantes; 5 ) influencia na mudança comportamental; 6) contribui para a identificação dos estudantes com o curso; 7) proporciona feedback espontâneo; 8) possibilita o acompanhamento da evolução do estudante no curso; e 9) estimula os alunos a concluir o curso em espaço de tempo menor (MAJURI; KOIVISTO; HAMARI, 2018).

Uma das mais reconhecidas limitações da educação a distância é a de que os estudantes, por se sentirem distantes dos professores, desejam a interação. E é exatamente isso que a gamificação possibilita. Os estudantes e os professores passam a ter um maior envolvimento. A ênfase nos aspectos lúdicos e o aprendizado ocorrendo de forma amigável torna os conhecimentos mais fáceis de serem lembrados e aplicados.

O termo gamificação evoca a ideia de jogos estruturados e tecnologias sofisticadas. De fato, existem plataformas que, mediante utilização da lógica da gamificação, possibilitam personalizar o ensino com base no desempenho dos estudantes ao longo do curso. Mas é possível utilizar a gamificação de forma simples para a obtenção de objetivos afetivos. $\mathrm{O}$ que importa é criar um ambiente de competição entre os estudantes, definir pontos para cada desempenho, acompanhar a classificação e premiar os que realizaram adequadamente as tarefas. 


\section{CONSIDERAÇÕES FINAIS}

Com as limitações impostas ao funcionamento das escolas em decorrência da pandemia da Covid-19, os professores do ensino superior - assim como os de outros níveis - passaram a adotar o ensino remoto como a principal estratégia para o alcance dos objetivos de aprendizagem. Não se trata, evidentemente, de estratégia capaz de suprir todas as necessidades dos estudantes, mas o que se tornou possível em decorrência da necessidade do distanciamento social.

Muitos professores procuraram ajustar-se à nova estratégia, mediante aulas expositivas transmitidas remotamente com o auxílio das plataformas, buscando aproximá-las, à medida do possível, às aulas presenciais. De fato, as plataformas, com os recursos de compartilhamento de transparências e textos escritos, bem como os fóruns e os chats, possibilitam o oferecimento de atividades didáticas adequadas para o alcance de muitos dos objetivos pretendidos pelos professores do ensino superior. É muito provável, após a pandemia, que as instituições desse nível de ensino passem a adotar - pelo menos parcialmente - o ensino remoto na maioria de seus cursos. Isso corresponderá à adoção do ensino híbrido como a forma preferencial de ensino superior.

Ocorre, porém, que uma das formas mais adotadas pelos professores no ensino remoto tem sido a videoconferência, já que é a que mais se aproxima das aulas expositivas, que ainda constituem uma das estratégias mais adotadas pelos docentes do ensino superior. Embora seja indiscutível seu valor pedagógico, sobretudo em relação ao alcance de objetivos cognitivos, a videoconferência mostra-se insuficiente em relação a objetivos de outros domínios, sobretudo do afetivo.

Torna-se necessário, portanto, que os professores do ensino superior se capacitem para utilizar os meios disponíveis para o ensino remoto também para o alcance de objetivos afetivos. Isso se torna possível, desde que se considerem as múltiplas estratégias de ensino grupal, assim como os métodos ativos, e que sejam associadas aos diversos recursos disponibilizados pelas plataformas de ensino remoto.

\section{REFERÊNCIAS}

AKOS, Patrick; KRETCHMAR, Jen. Investigating grit at a non-cognitive predictor of college success. The Review of Higher Education, Baltimore, v. 40, n. 2, p. 163-186, 2017. Disponível em: https://muse.jhu.edu/article/640608. Acesso em: 06 ago. 2020. DOI:

https://doi.org/10.1353/rhe.2017.0000.

ANDERSON, Lorin W. et al. A taxonomy for learning, teaching, and assessing: A revision of Bloom's taxonomy of educational objectives. Essex: Longman, 2001. 
BAKER, Ryan Shaun J. D. et al. Better to be frustrated than bored: The incidence, persistence, and impact of learners' cognitive-affective states during interactions with three different computer-based learning environments. International Journal of Human-Computer Studies, Amsterdã, v. 68, n. 4, p. 223-241, 2010. Disponível em:

https://www.sciencedirect.com/science/article/abs/pii/S1071581909001797. Acesso em: 06 ago. 2020. DOI: https://doi.org/10.1016/j.ijhcs.2009.12.003.

BERGMANN, Jonathan. Sala de aula invertida. São Paulo: Grupo Gen-LTC, 2016.

BIRBECK, David. Graduate qualities and the affective domain: New horizons to explore. 2009. Tese (Doutorado em Educação) - Learning and Teaching Unit, University of South Australia, Adelaide, 2009.

BLOOM, Benjamin Samuel et al. Taxonomy of educational objectives: the classification of educational goals: handbook I: cognitive domain. Nova lorque: D. Mckay, 1956.

BOBBITT, John Franklin. How to Make a Curriculum. Boston: Houghton Mifflin, 1924.

BOLIN, Aaron U.; KHRAMTSOVA, Irina; SAARNIO, David. Using student journals to stimulate authentic learning: balancing Bloom's cognitive and affective domains. Teaching of

Psychology, Oxfordshire, v. 32, n. 3, p. 154-159, 2005. Disponível em:

https://www.tandfonline.com/doi/abs/10.1207/s15328023top3203_3. Acesso em: 06 ago. 2020. DOI: https://doi.org/10.1207/s15328023top3203_3.

CATE, Olle Ten; DE HAES, Hanekke (JCJM). Summative assessment of medical students in the affective domain. Medical Teacher, [S.I], v. 22, n. 1, p. 40-43, 2000. DOI:

https://doi.org/10.1080/01421590078805.

CLEVELAND-INNES, Martha; ALLY, Mohamed. Learning to feel: Education, affective outcomes and the use of online teaching and learning. European Journal of Open, Distance and Elearning, Berlim, v. 10, n. 2, p. 1-17, 2007. Disponível em:

https://www.eurodl.org/?p=archives\&year=2007\&halfyear=2\&..\&abstract=285. Acesso em: 06 ago. 2020.

D'MELLO, Sidney; GRAESSER, Art. Dynamics of affective states during complex learning. Learning and Instruction, Amsterdã, v. 22, n. 2, 145-157, 2012. Disponível em: https://www.sciencedirect.com/science/article/abs/pii/S0959475211000806. Acesso em: 29 set. 2020. DOI: https://doi.org/10.1016/j.learninstruc.2011.10.001.

DU BOULAY, Bento. Projetando interação educacional afetiva e cognitiva. In: LUCKIN, Rosemary et al. Handbook of Design in Educational Technology. Nova lorque: Routledge, 2013. p. 168-175. Disponível em: encurtador.com.br/Fagms. Acesso em: 06 ago. 2020.

ECCLESTONE, Kathryn; HAYES, Dennis. Afetar: conhecimento, comunicação, criatividade e emoção. 2008. Disponível em:

https://www.researchgate.net/publication/265454681_The_Dangerous_Rise_of_Therapeuti c_Education. Acesso em: 06 ago 2020. DOI: https://doi.org/10.4324/9780203870563. 
FALOUT, Joseph; ELWOOD, James; HOOD, Michael. Demotivation: Affective states and learning outcomes. System, Amsterdã, v. 37, n. 3, p. 403-417, 2009. Disponível em: https://www.sciencedirect.com/science/article/abs/pii/S0346251X09000566. Acesso em: 06 ago 2020. DOI: https://doi.org/10.1016/j.system.2009.03.004.

FASSO, Wendy; KNIGHT, Bruce Allen. Afetam no aprendizado on-line: resultados, emoções e estados afetivos. In: Aplicações Inovadoras de Pedagogia Online e Design de Cursos.

Hershey: IGI Global, 2018. p. 128-153.

GIL, Antonio Carlos. Metodologia do ensino superior. São Paulo: Atlas, 2020.

GREENWOOD, Charles R.; CARTA, Judith J.; HALL, R. Vancel. The Use of Peer Tutoring Strategies in Classroom Management and Educational Instruction, School Psychology Review, Londres, v. 17, n. 2, 258-275, 1988. DOI: https://doi.org/10.1080/02796015.1988.12085342.

GRIFFIN, Lynda; ROY, James. A great resource that should be utilised more, but also a place of anxiety: student perspectives on using an online discussion forum. Open Learning: The Journal of Open, Distance and e-Learning, Londres, p. 1-16, 2019. DOI:

https://doi.org/10.1080/02680513.2019.1644159.

GRONLUND, Norman E. How to write and use instructional objectives. Englewood Cliffs: Merrill/Prentice-Hall, 1995.

HOMER, Bruce D.; PLASS, Jan L.; BLAKE, Linda. The effects of video on cognitive load and social presence in multimedia-learning. Computers in Human Behavior, Amsterdã, v. 24, n. 3, 786-797, 2008. Disponível em:

https://www.sciencedirect.com/science/article/abs/pii/S0747563207000544. Acesso em: 29 set. 2020. DOI: https://doi.org/10.1016/j.chb.2007.02.009.

HORN, Michael B.; STAKER, Heather. Blended: usando a inovação disruptiva para aprimorar a educação. Porto Alegre: Penso, 2015.

JAGGER, Suzy. Affective learning and the classroom debate. Innovations in Education and Teaching International, Londres, v. 50, n. 1, p. 38-50, 2013. Disponível em: https://srhe.tandfonline.com/doi/abs/10.1080/14703297.2012.746515. Acesso em: 06 ago. 2020. DOI: https://doi.org/10.1080/14703297.2012.746515.

JOHNSON, David W.; JOHNSON, Roger T. Fazendo funcionar a aprendizagem cooperativa. Teoria em prática, [S.I.], v. 38, n. 2, p. 67-73, 1999.

KRATHWOHL, David R.; BLOOM, Benjamin S.; MASIA, Bertram B. Taxonomy of educational objectives, handbook ii: affective domain. Nova Iorque: David McKay Company, 1964.

LEE, Cheng-Yuan. Student motivation in the online learning environment. Journal of Educational Media \& Library Sciences, Taiwan, v. 37, n. 4, p. 367-375, 2000. Disponível em: http://joemls.dils.tku.edu.tw/fulltext/37/37-4/367-375.pdf. Acesso em: 06 ago. 2020. 
LYNCH, Sandra; HART, Bethne; COSTA, Catherine M. Giving voice to values: An undergraduate nursing curriculum project. Collegian, Palo Alto, v. 21, n. 4, p. 367-373, 2014. Disponível em:

https://www.sciencedirect.com/science/article/abs/pii/S1322769613000929. Acesso em: 06 ago. 2020. DOI: https://doi.org/10.1016/j.colegn.2013.09.004.

MAGER, Robert F. Preparing instructional objectives. Palo Alto: Fearon Publishers, 1962. Disponível em: https://eric.ed.gov/?id=ED018143. Acesso em: 29 set. 2020.

MAJURI, Jenni; KOIVISTO, Jonna; HAMARI, Juho. Gamification of education and learning: A review of empirical literature. In: GAMIFIN 2018, 2., 2018. Pori. Proceedings of the 2nd international GamiFIN conference. Pori: CEUR-WS, 2018. p. 11-19. Disponível em:

https://trepo.tuni.fi/bitstream/handle/10024/104598/gamification_of_education_2018.pdf. Acesso em: 29 set. 2020.

MARTIN, Barbara L.; BRIGGS, Leslie J. The affective and cognitive domains: Integration for instruction and research. Nova Jérsei, Educational Technology, 1986. Disponível em: encurtador.com.br/jtFIU. Acesso em: 06 ago. 2020.

MARTIN, Florence; PARKER, Michele A. Use of synchronous virtual classrooms: Why, who, and how. MERLOT Journal of Online Learning and Teaching, Long Beach, v. 10, n. 2, p. 192210, 2014.

MOORE, Schuster M. Theory of transactional distance. In: KEEGAN, Desmond. (ed.), Theoretical principles of distance education. Nova lorque: Routledge, 1997. p. 22-38.

MOORE, Michael G.; KEARSLEY, Gres. Educação a distância: sistemas de aprendizagem online. São Paulo: Cengage Learning, 2013.

MORENO, Jacob Levy. Fundamentos do psicodrama. São Paulo: Summus, 1983.

NORTHEY, Gavin et al. Increasing student engagement using asynchronous learning. Journal of Marketing Education, Londres, v. 37, n. 3, p. 171-180, 2015. DOI:

https://doi.org/10.1177/0273475315589814.

OLATUNJI, Michael Olalekan. Online Education: Issues, Challenges and Implications. Khazar Journal of Humanities \& Social Sciences, Baku, v. 16, n. 3, 2013. Disponível em: https://jhsskhazar.org/wp-

content/uploads/2010/04/0005KHAZAR_Journal_of_Humanities_and_Social_Sci_ences1.pdf . Acesso em: 06 ago. 2020.

SEELS, Barbara; GLASGOW, Zita. Exercises in instructional design. Columbus: Merrill Publishing Company, 1990.

SHOFFNER, Melanie. The place of the personal: Exploring the affective domain through reflection in teacher preparation. Teaching and Teacher Education, Amsterdã, v. 25, n. 6, p. 783-789, 2009. Disponível em: 
https://www.sciencedirect.com/science/article/abs/pii/S0742051X08002011. Acesso em: 06 ago 2020. DOI: https://doi.org/10.1016/j.tate.2008.11.012.

SHORT, John; WILLIAMS, Ederyn; CHRISTIE, Bruce. The social psychology of telecommunications. [S.I.]: John Wiley \& Sons, 1976.

SNELSON, Chareen; ELISON-BOWERS, Patt R. Using YouTube videos to engage the affective domain in e-learning. In: MENDEZ-VILAS; Antonio et al. Research, Reflections and Innovations in Integrating ICT in Education. Badajoz: Formatex, 2009. p. 1481-1485. Disponível em: https://scholarworks.boisestate.edu/edtech_facpubs/50/. Acesso em: 06 ago. 2020.

TYLER, Ralph W. Basic principles of curriculum and instruction. Chicago: University of Chicago Press, 1949.

VAN DER HOEVEN KRAFT, Katrien J. et al. Engaging students to learn through the affective domain: A new framework for teaching in the geosciences. Journal of Geoscience Education, Northfield, v. 59, n. 2, p. 71-84, 2011. Disponível em:

https://www.tandfonline.com/doi/abs/10.5408/1.3543934a. Acesso em: 06 ago. 2020. DOI: https://doi.org/10.5408/1.3543934a.

WHITE, Karl R. T-groups revisited: Self-concept change and the "Fish-Bowling" technique. Small Group Behavior, Pensilvânia, v. 5, n. 4, p. 473-485, 1974. DOI:

https://doi.org/10.1177/104649647400500407.

\section{Antonio Carlos Gil}

Doutor em Saúde Pública pela USP (1998), doutor em Ciências Sociais pela FESPSP (1981), mestre em Ciências Sociais pela FESPSP (1978), licenciado em Pedagogia pela FFCL Professor Carlos Pasquale (1978), bacharel em Ciências Políticas e Sociais pela USCS (1971), docente dos Programas de Mestrado em Administração e Educação e Doutorado em Administração da USCS.

acgil@uol.com.br

\section{Arquimedes Pessoni}

Pós-doutor em Medicina na Faculdade de Medicina do ABC (linha de pesquisa em Educação na Saúde/Saúde Coletiva-2014). Possui graduação em Jornalismo pela UMESP (1984), mestrado em Comunicação Social pela UMESP (2002) e doutorado em Comunicação Social pela UMESP (2005). Docente dos Programas de Mestrado em Inovação na Comunicação de Interesse Público e Inovação no Ensino Superior em Saúde da USCS.

arquimedes.pessoni@uol.com.br 